\title{
MONITORAMENTO DO MESOTELIOMA NO SUL DO BRASIL: UMA REALIDADE AINDA A SER ESTUDADA*
}

\author{
Francisco José Koller ${ }^{1}$, Leila Maria Mansano Sarquis², Maria de Fátima Mantovani², Fernanda Moura \\ D’Almeida Miranda ${ }^{3}$, Dario Consonni ${ }^{4}$, Carolina Mensi ${ }^{5}$
}

\begin{abstract}
RESUMO: O Brasil é um dos maiores produtores de crisotila do mundo, mas a ocorrência de mesotelioma é aparentemente baixa. No entanto, a identificação dos casos torna-se difícil por causa de erros de diagnóstico e registro de morte. Este é um estudo epidemiológico, descritivo e retrospectivo de registros hospitalares do Instituto Nacional de Câncer do Brasil, foram selecionados registros câncer de pleura ou histologia do mesotelioma entre 2001-2014. Foram identificados 208 registros, entretanto apenas 58 casos com histologia de mesotelioma. Notavelmente, $13(22 \%)$ tinham sido codificados incorretamente como câncer de pulmão. A última atividade laboral estava ausente em metade dos casos. Concluiu-se que os dados hospitalares existentes em relação ao mesotelioma ainda são inadequados por imprecisão e codificação errônea. Além disso, nos registros ainda não existem informações do local de trabalho, bem como da ocupação. Estes achados confirmam a necessidade de um registro de casos de mesotelioma.
\end{abstract}

DESCRITORES: Mesotelioma; Amianto; Epidemiologia.

\section{MONITORING OF MESOTHELIOMA IN SOUTHERN BRAZIL: A SITUATION TO BE INVESTIGATED}

\begin{abstract}
Brazil is one of the world's greatest producers of chrysotile, but the occurrence of mesothelioma is apparently low. However, identification of cases of mesothelioma is difficult due to errors of diagnosis and registries of deaths. This is an epidemiological, descriptive and retrospective study of hospital records of Brazil's National Institute of Cancer (INCA). Records of pleural cancer or mesothelioma histology from the 2001-2014 period were selected. We identified 208 records, but only 58 cases of mesothelioma histology. Notably, $13(22 \%)$ had been incorrectly coded as lung cancer. In half the cases, there was no registration of the latest occupations of the patients. We conclude that hospital data on mesothelioma is still imprecise and was incorrectly coded. Besides, the registries do not include information on the workplace and occupation. These findings confirm the need for a proper registry of cases of mesothelioma.
\end{abstract}

DESCRIPTORS: Mesothelioma; Asbestos; Epidemiology.

\section{INSPECCIÓN DEL MESOTELIOMA EN SUR DE BRASIL: UNA REALIDAD QUE TODAVÍA DEBE SER ESTUDIADA}

RESUMEN: Mientras se apunta que Brasil es un de los mayores productores de crisotila en el mundo, la ocurrencia de mesotelioma es aparentemente baja. Sin embargo, la identificación de los casos es dificil a causa de errores de diagnóstico y registro de muerte. Este es un estudio epidemiológico, descriptivo y retrospectivo de registros hospitalarios del Instituto Nacional de Cáncer de Brasil. Fueron seleccionados registros de cáncer de pleura o histología del mesotelioma entre 2001 y 2014 . Se identificaron 208 registros, pero solamente 58 casos con histología de mesotelioma. Se constató que 13 (22\%) fueron codificados de modo incorrecto como cáncer de pulmón. La última actividad laboral no estaba presente en mitad de los casos. Se concluye que los datos hospitalarios existentes acerca del mesotelioma todavía son inadecuados por imprecisión y codificación errónea. Además, en los registros no hay informaciones del local de trabajo, así como de la ocupación del paciente. Estos hallados confirman la necesidad de un registro de casos de mesotelioma.

DESCRIPTORES: Mesotelioma; Asbesto; Epidemiología.

* Artigo extraído da dissertação intitulada: "Investigação das neoplasias malignas de brônquio, de pulmão e de pleura no sul do Brasil". Universidade Federal do Paraná, 2015.

${ }^{1}$ Enfermeiro. Mestre em Enfermagem. Docente da Faculdade Integrada Santa Cruz. Curitiba, PR, Brasil

${ }^{2}$ Enfermeira. Doutora em Enfermagem. Docente de Graduação e Pós Graduação de Enfermagem da Universidade Federal do Paraná. Curitiba, PR, Brasil

${ }^{3}$ Enfermeira. Doutora em Enfermagem. Coordenadora do Curso de Pós Graduação em Enfermagem do Trabalho da Universidade Positivo. Curitiba, PR, Brasil

${ }^{4}$ Médico. Doutor em Medicina do Trabalho. Docente da Universidade de Milão. Milão - Itália. Unidade de Epidemiologia Fondazione IRCCS Ca' Granda Ospedale Maggiore Policlinico, Milão - Itália.

${ }^{5}$ Bióloga. Doutora em Saúde Pública. Epidemiologista do Registro de Mesotelioma de Lombardia. Milão - Itália. Unidade de Epidemiologia. Fondazione IRCCS $\mathrm{Ca}^{\prime}$ Granda Ospedale Maggiore Policlinico, Milão - Itália.

Autor Correspondente:

Recebido: 07/11/2016

Francisco José Koller

Universidade Federal do Paraná

Finalizado: 20/12/2016

Av. Prefeito Lothário Meissner, 632 - 80210-170 - Curitiba, PR, Brasil

E-mail: enfkoller@yahoo.com.br 


\section{INTRODUÇÃO}

O mesotelioma é um dos tipos de câncer mais letais, com incidência crescente em todo o mundo, com necessidade de monitorar a incidência, identificar fontes de exposição ao amianto, prestar assistência médico-legal aos pacientes e seus familiares e prever as tendências futuras de incidência ${ }^{(1-2)}$. A primeira descrição completa foi feita em 1767 por Joseph Lieutaud, entretanto em 1931 houve a designação do termo "mesotelioma" por Klempere e Rabim para alguns tumores de pleura ${ }^{(3)}$. Trata-se de uma neoplasia proveniente das células mesoteliais e submesoteliais que compõem as camadas pleurais, o pericárdio, o peritônio e a túnica vaginal do testículo, cuja característica da patologia é agressiva, sem cura, com período de latência muito longo após exposição ao fator etiológico ${ }^{(4)}$.

Uma das principais causas para o desenvolvimento do câncer é a inalação de fibras de amianto(5). A literatura afirma que cerca de $90 \%$ dos casos de mesotelioma estão relacionados à exposição a este mineral, sendo que na maioria dos casos o contato com a fibra ocorre no ambiente de trabalho ${ }^{(1-6)}$.

O Brasil é o terceiro maior produtor mundial de amianto, com baixa tendência sobre as políticas do banimento deste mineral na cadeia industrial ${ }^{(7)}$.

Em 2010, cerca de 300.000 trabalhadores estavam expostos ao amianto e cerca de 20.000 encontravamse na indústria primária, como oficinas de freios e embreagens e da construção civil. Estes trabalhadores não eram acompanhados pelo serviço de saúde ocupacional, fato este que torna uma preocupação frente a uma exposição não visível, consequentemente não monitorada ${ }^{(2)}$.

A produção brasileira do amianto utiliza $40 \%$ no mercado interno, principalmente no estado do Paraná, São Paulo, Rio de Janeiro, Goiás, Rio Grande do Sul, Minas Gerais, Bahia, Santa Catarina e Alagoas, e uma importação de 44 mil toneladas de crisótila da a China, a serem empregadas em artefatos de fibrocimento, aquisição e reforma de equipamentos, inovações tecnológicas e de sistemas, infraestrutura, meio ambiente e saúde e segurança no trabalho( ${ }^{(8)}$.

As manifestações clínicas do mesotelioma pleural são tosse seca, dispneia, dor torácica, emagrecimento importante em curto período de tempo, fraqueza e derrame pleural com característica hemorrágica. Em função de metástases, reações leucemoides e anormalidade metabólicas ${ }^{(9)}$ e pode ocorrer até 30 anos após a exposição às fibras do amianto, sendo três vezes mais suscetível em homens do que em mulheres, com aumento no número de adoecidos após os 60 anos de idade ${ }^{(10)}$.

O diagnóstico patológico do mesotelioma é notoriamente difícil. A sobrevida mediana é aproximadamente entre 08 a 12 meses e o paciente geralmente apresenta sinais e sintomas de depleção clínica acentuada, dificultando a realização dos procedimentos invasivos ${ }^{(11)}$.

Diante deste cenário, o objetivo deste estudo foi descrever os casos de mesotelioma no Sul do Brasil registrado no sistema no Registro Hospitalar de Câncer.

\section{MÉTODO}

Trata-se de um estudo epidemiológico, descritivo e retrospectivo, realizado com os dados do Integrador Registro Hospitalar do Câncer (IRHC) do Sistema para Informatização dos Dados de Registros Hospitalares de Câncer, disponibilizado pelo Instituto Nacional do Câncer José Alencar da Silva - Brasil.

No período de janeiro de 2001 a dezembro de 2014 (14 anos), foram extraídos os casos com ICD10=C38.4 (Câncer de Pleura) e C34 (Câncer de Pulmão) para as morfologias de mesotelioma buscando a respectiva característica (9053/3: bifásica; 9052/3: epitelioide; 9051/3: fibroso e 9050/3 não especificado).

As variáveis eram demográficas (sexo, idade, raça, nível de instrução e ocupação) e clínicas (tipo morfológico, localização do tumor, estadiamento, tratamento realizado e estado final após o primeiro tratamento realizado).

Este estudo está em conformidade com os preceitos éticos e foi aprovado sob Parecer: 677.015 de 29/05/2014, conforme Resolução n466/12. 
Foram extraídos 208 casos, 194 (93,2\%) classificados como câncer de pleura e 14 (6,8\%) como câncer de pulmão. Foram excluídos os registros não relacionados ao mesotelioma, sendo 26 (12,5\%) adenocarcinomas, $20(9,6 \%)$ linfomas, $07(3,4 \%)$ carcinomas, $08(3,8 \%)$ sarcomas, $02(1 \%)$ mielomas e $12(5,8 \%)$ com outras morfologias. Também foram excluídos 75 registros com definição genérica de "neoplasia maligna". A amostra final de mesotelioma resultou em 58 (27,9\%) casos: 10 (17,2\%) epitelioides, $5(8,6 \%)$ fibrosos, $1(1,7 \%)$ bifásico, enquanto na maioria, 42 (72,4\%) a morfologia era de tipo não especificado (Tabela 1).

Tabela 1- Caracterização dos casos de mesotelioma, segundo sexo, faixa etária e morfologia. Curitiba, PR, Brasil, 2014.

\begin{tabular}{|c|c|c|c|c|c|c|c|}
\hline \multirow[t]{2}{*}{ CARACTERÍSTICA } & \multirow[t]{2}{*}{ VARIÁVEL } & \multicolumn{2}{|c|}{ PARANÁ } & \multicolumn{2}{|c|}{$\begin{array}{l}\text { RIO GRANDE DO } \\
\text { SUL }\end{array}$} & \multicolumn{2}{|c|}{ SANTA CATARINA } \\
\hline & & N. & $\%$ & N. & $\%$ & N. & $\%$ \\
\hline \multirow[t]{2}{*}{ SEXO } & Feminino & 5 & 33,3 & 5 & 17,8 & 7 & 46,6 \\
\hline & Masculino & 10 & 66,7 & 23 & 82,2 & 8 & 53,4 \\
\hline \multirow[t]{4}{*}{ FAIXA ETÁRIA } & Antes dos 49 anos & 3 & 20 & 9 & 32,3 & 4 & 26,7 \\
\hline & Dos 50 a 59 anos & 4 & 26,7 & 8 & 28,5 & 3 & 20 \\
\hline & Dos 60 a 69 anos & 5 & 33,3 & 4 & 14,2 & 4 & 26,7 \\
\hline & Acima dos 70 anos & 3 & 20 & 7 & 25 & 4 & 27,6 \\
\hline \multirow[t]{4}{*}{ MORFOLOGIA } & Mesotelioma bifásico & 0 & 0 & 1 & 3,6 & 0 & 0 \\
\hline & Mesotelioma epitelioide & 4 & 26,6 & 5 & 17,8 & 1 & 6,6 \\
\hline & Mesotelioma fibroso & 2 & 13,4 & 2 & 7,2 & 1 & 6,7 \\
\hline & $\begin{array}{l}\text { Mesotelioma não } \\
\text { especificado }\end{array}$ & 9 & 60 & 20 & 71,4 & 13 & 86,7 \\
\hline
\end{tabular}

Fonte: IRHC/SIDRH/INCA (2016)

A maioria dos casos era do Rio Grande do Sul. As atividades ocupacionais realizadas nos últimos três meses foram encontradas em 29 casos (50\%) nos seguintes setores: construção civil, metalúrgica, transportes e agricultura.

A principal descrição dos casos foi com idade média de 58 anos, sexo masculino 41 (70,7\%), da raça branca $48(82,8 \%)$, casados $32(55,2 \%)$ e com ensino fundamental completo $15(25,9 \%)$. Em relação à característica do mesotelioma, foram registrados principalmente com estádio IV 18 (31\%), que fizeram como primeira opção de tratamento a quimioterapia $18(31 \%)$, entretanto $12(20,7 \%)$ registros não fizeram nenhum tratamento.

\section{- DISCUSSÃO}

No Brasil, as pesquisas epidemiológicas sobre o mesotelioma são escassas ${ }^{(12)}$. Isto provavelmente ocorre devido ao baixo número de casos notificados nos sistemas de informação em saúde disponíveis no território nacional. Considera-se necessário o aprimoramento correto do IRHC, pelo fato deste sistema ser uma das fontes de informação que possibilita a avaliação da qualidade da atenção ao paciente oncológico, da taxa de sobrevida e dos fenômenos temporais associados ao câncer ${ }^{(13)}$. No entanto, a falta de registros dos dados nos prontuários dos pacientes acarreta uma fragilidade na qualidade dos IRHC, negligenciando o acesso das informações sobre o paciente, do diagnóstico, da caracterização do tumor, do tipo de tratamento realizado, bem como da evolução do paciente ${ }^{(14)}$.

Uma situação a ser analisada na codificação do mesotelioma no sistema IRHC é a utilização da Classificação Internacional de Doenças CID, pois até 1995 o mesotelioma de pleura era classificado como câncer de pleura pelo código 163 da $9^{a}$ Revisão CID-9. Com vistas a permitir uma classificação 
mais elucidativa, a 10 ${ }^{\mathrm{a}}$ Revisão da CID, adotada pelo Brasil em 1996, destinou um código específico para o mesotelioma, atribuindo o código C45, com especificadores C45.0 (mesotelioma de pleura), C45.1 (mesotelioma de peritônio), C45.2 (mesotelioma de pericárdio), C45.7 (mesotelioma de outras localizações) e C45.9 (mesotelioma não especificado) ${ }^{(15)}$.

Entretanto nesse tipo de sistema de informação utiliza-se uma edição da Classificação Internacional de Doenças para Oncologia - a CID-O/3 para especificar a localização e a morfologia do tumor e as posteriores tabulações e seleção de casos para os estudos clínicos e epidemiológicos, incluindo os comparativos temporais e interinstitucionais, categorizando os casos de mesotelioma de pleura pela localização C38.4 e atribuindo códigos morfológicos ${ }^{(16)}$.

Para os casos registrados com base na CID-10, estudos têm destacado que essa correspondência tem permanecido, de forma que casos com morfologia de mesotelioma maligno ainda estão sendo notificados como câncer de pleura, por meio do código C38.4, em vez de serem C45, o que tem recebido o nome de "fuga" ou "escape". Por ser reconhecido como um problema histórico, países como a Grã-Bretanha têm monitorado anualmente os índices de "escape" do mesotelioma ${ }^{(17)}$.

Um estudo realizado no Brasil sobre a mortalidade por mesotelioma apresentou a análise de óbitos entre janeiro de 2000 e dezembro de 2012, que registrou 929 e 1379 registros de óbitos de C45 e C38.4 respectivamente ${ }^{(2)}$.

O mesotelioma é um tipo de câncer causado pela exposição das pessoas à fibra do amianto. Em muitos países o mesotelioma está aumentando mesmo após a proibição da comercialização e manipulação desta mineral em mais de 60 países $^{(15)}$.

Nos países desenvolvidos as incidências são altas e existem políticas de rastreamento e sistemas de qualidade para controle dos $\operatorname{casos}^{(1819)}$.

Em comparação com outros países, no Brasil evidencia-se um atraso de 15 a 20 anos sobre a mortalidade do mesotelioma, o que é consistente com o consumo de amianto no país. Alguns pesquisadores estão estudando esta temática e futuros estudos mostrarão a magnitude desta problemática com resultados ainda a serem apresentados ${ }^{(2)}$.

Um estudo realizado no Rio de Janeiro, no período entre 1979 a 2000 com 83 casos de mesotelioma apresentou uma razão homem/mulher de 2:1 relacionados à exposição ao amianto (20). Em Santa Catarina, verificou-se 30 óbitos por mesotelioma ocorridos no período de 1998 a 2009, que apontaram o mesotelioma de pleura em trabalhadores das forças armadas, policiais e bombeiros militares e nos trabalhadores de serviços de reparos e manutenção ${ }^{(21)}$. No Rio Grande do Sul, entre 1999 a 2003, foram 25 óbitos por mesotelioma principalmente em aposentados, agricultores e doméstica/do lar ${ }^{(22)}$.

Nesta pesquisa, foi observada a falta da identificação da atividade laboral em um terço dos casos. O estudo realizado em 2008 no Estado do Rio de Janeiro foi similar, foram analisados 221 prontuários de pacientes. Concluiu-se que houve uma fragilidade no registro da ocupação no momento de admissão do paciente, demonstrando que $45 \%$ das informações sobre ocupação estavam em branco ou foram preenchidas de maneira incompleta, dificultando a análise etiológica do câncer ${ }^{(13)}$.

Embora essa informação estivesse completa, mesmo assim não seria útil, pois este registro é apenas dos últimos três meses de atividade laboral. Como a latência do mesotelioma é muito longa, faz-se necessário investigar todo o período de atividade laboral das pessoas.

A utilização destes resultados visa uma avaliação da qualidade da assistência ao paciente portador de mesotelioma e necessita de sensibilização dos profissionais e dos gestores das unidades hospitalares para que essas informações sejam preenchidas corretamente nos prontuários, já que a falta de preenchimento de dados prejudica a análise das variáveis para a obtenção de informações sobre o paciente, o diagnóstico, a caracterização do tumor, o tratamento, a evolução do paciente e relativa ao óbito ${ }^{(23)}$.

Houve limitações neste estudo e destacamos que este registro de dados foi insuficiente para extrair corretamente os casos de mesotelioma, bem como informações sobre o tipo histológico e o tipo de atividade laboral completa. O IRHC não tem características para captar validamente os casos deste tipo de câncer. 
Frente à incompletude de dados por este instrumento ser inadequado para o monitoramento do mesotelioma, torna-se necessário a implantação de um registro dedicado aos casos desta doença. Para cada caso confirmado, deveriam ser registradas as informações sobre a história laboral completa, estimulando por meio das ações de vigilância a produção do conhecimento necessário sobre os riscos causados por este mineral. Assim, fomentar as ações das autoridades nacionais de saúde pública e organizações sociais para o banimento do amianto e da assistência ao paciente e familiares com mesotelioma.

\section{REFERÊNCIAS}

1. Mensi C, de Matteis S, Dallari B, Riboldi L, Bertazzi PA, Consonni D. Incidence of mesothelioma in Lombardy, Italy: exposure to asbestos, time patterns and future projections. Occup. Environ. Med. [Internet] 2016;73(9) [acesso em 07 dez 2016]. Disponível: http://dx.doi.org//10.1136/oemed-2016-103652.

2. Algranti E, Saito CA, Carneiro APS, Moreira B, Mendonça EMC, Bussacos MA. The next mesothelioma wave: mortality trends and forecast to 2030 in Brazil. Cancer Epidemiol. [Internet] 2015;39(5) [acesso em 30 nov 2016]. Disponível: http://dx.doi.org/10.1016/j.canep.2015.08.007.

3. Roe OD, Stella GM. Malignant pleural mesothelioma: history, controversy and future of a manmade epidemic. Eur Respir Rev. [Internet] 2015;24(135) [acesso em 30 nov 2016]. Disponível: http://dx.doi. org//10.1183/09059180.00007014.

4. Neumann V, Löseke S, Nowak D, Herth FJF, Tannapfel A. Malignant pleural mesothelioma: incidence, etiology, diagnosis, treatment, and occupational health. Dtsch Arztebl Int. [Internet] 2013;110(18) [acesso em 05 dez 2016]. Disponível: http://dx.doi.org/10.3238/arztebl.2013.0319.

5. International Agency for Research on Cancer. Asbestos, (chrysotile, amosite, crocidolite, tremolite, actinolite, and anthophyllite). In: Metals, arsenic, dusts and fibres. A review of human carcinogens. Lyon: IARC (Monograph on the Evaluation of Carcinogenic Risks to Humans vol. 100C) [Internet] 2012 [acesso em 07 dez 2016]. Disponível: http://monographs.iarc.fr/ENG/Monographs/vol100C/mono100C.pdf.

6. Pawełczyk A, Božek F. Health risk associated with airborne asbestos. Environ Monit Assess. [Internet] 2015;(187) [acesso em 25 nov 2016]. Disponível: http://dx.doi.org//10.1007/s10661-015-4614-3.

7. Kazan-Allen L. International Ban Asbestos Secretariat. Current asbestos bans and restrictions. [Internet] 2014 [acesso em 30 nov 2016]. Disponível: http://ibasecretariat.org/asbestos_ban_list.php.

8. Brasil. Departamento Nacional de Produção Mineral. Sumário Mineral. [Internet] Brasília: Departamento Nacional de Produção Mineral; 2016 [acesso em 30 nov 2016]. Disponível: http://www.dnpm.gov.br/dnpm/ sumarios/sumario-mineral-2015.

9. Ahmed I, Tipu SA, Ishtiaq S. Malignant mesothelioma. Pak. J. Med. Sci. [Internet] 2013;29(6) [acesso em 05 dez 2011]. Disponível: http://dx.doi.org/10.12669/pjms.296.3938.

10. McCormack V, Peto J, Byrnes G, Straif K, Boffetta P. Estimating the asbestos-related lung cancer burden from mesothelioma mortality. Br J Cancer. [Internet] 2012;106(3) [acesso em 25 nov 2016]. Disponível: http://dx.doi. org//10.1038/bjc.2011.563.

11. Leong SL, Gary Lee SG, Robinson BW, Creaney J. Role of Mesothelin Measurement in Patients With CytologyNegative Pleural Effusion Undergoing Medical Thoracoscopy. PLEURA. [Internet] 2015 [acesso em 30 nov 2016]. Disponível: http://dx.doi.org//10.1177/2373997515579639.

12. Forouzanfar MH, Alexander L, Anderson HR, Bachman VF, Biryukov S, Brauer $\mathrm{S}$ et al. Global, regional, and national comparative risk assessment of 79 behavioural, environmental and occupational, and metabolic risks or clusters of risks in 188 countries, 1990-2013: a systematic analysis for the Global Burden of Disease Study 2013. Lancet. [Internet] 2015;386(10010) [acesso em 07 dez 2016]. Disponível: http://dx.doi.org/10.1016/S01406736(15)00128-2. 
13. Tabalipa MM, Boccolini PMM, Simões DR, Chrisman JR, Otero UB, Garbin HBR, et al. Informação sobre ocupação em registros hospitalares de câncer no estado do Rio de Janeiro. Cad. saúde colet. [Internet] 2011;19(3) [acesso em 07 dez 2016]. Disponível: http://www.iesc.ufrj.br/cadernos/images/csc/2011_3/artigos/csc_v19n3_278286.pdf.

14. Branco BPC, Carvalho FA, Paiva FA, Branco ITC. Dificuldades no diagnóstico diferencial entre mesotelioma pleural e adenocarcinoma metastático para pleura patológica. Jornal Brasileiro de Pneumologia. [Internet] 2013;101(6) [acesso em 05 dez 2016]. Disponível: http://files.bvs.br/upload/S/0047-2077/2013/v101n6/a4016.pdf.

15. Stayner L, Welch LS, Lemen R. The worldwide pandemic of asbestos-related diseases. Annu Rev Public Health. [Internet] 2013;(34) [acesso em 25 nov 2016] Disponível: http://dx.doi.org//10.1146/annurevpublhealth-031811-124704.

16. Ministério da Saúde (BR). Instituto Nacional do Câncer José de Alencar Gomes da Silva (INCA). Registro Hospitalares de Câncer: Planejamento e Gestão. Rio de Janeiro: Ministério da Saúde; Inca; 2010.

17. Pedra F, da Silva PO, Mattos IE, Castro HA. Mortalidade por Mesotelioma no Brasil, 1980 a 2010 / Mortalidade por Mesotelioma in Brasil, 1980 a 2010. Rev. Bras. Cancerol. [Internet] 2014;60(3) [acesso em 30 nov 2016]. Disponível: http://www1.inca.gov.br/rbc/n_60/v03/pdf/03-artigo-mesothelioma-mortality-rate-in-brazil-1980-to-2010.pdf.

18. Park EK, Takahashi K, Hoshuyama T, Cheng TJ, Delgermaa V, Le GV, et al. Global magnitude of reported and unreported mesothelioma. Environ Health Perspect. [Internet] 2011;119(4) [acesso em 05 nov 2016]. Disponível: http://dx.doi.org/10.1289/ehp.1002845.

19. Delgermaa V, Takahashi K, Park EK, Vinh Le G, Hara T, Sorahan T. Global mesothelioma deaths reported to the World Health Organization between 1994 and 2008. Bull World Health Organ. [Internet] 2011;89(10) [acesso em 30 nov 2016]. Disponível: http://dx.doi.org/10.2471/BLT.11.086678.

20. Pinheiro GA, Antao VCS, Monteiro MMT, Capelozzi VL, Terra-Filho M. Mortality from pleural mesothelioma in Rio de Janeiro, Brazil, 1979-2000: Estimation from death certificates, hospital records, and histopathologic assessments. Int. J. Occup. Environ. Health. [Internet] 2003;9(2) [acesso em 30 nov 2016]. Disponível: http://dx.doi. org/10.1179/oeh.2003.9.2.147.

21. Mattos GR, Pinheiro RDC. Perfil dos óbitos por mesotelioma registrados no sistema de informação de mortalidade em Santa Catarina, no período de 1998-2009. Rev. Saúde Pública. [Internet] 2011;4(1) [acesso em 26 nov 2016]. Disponível: http://esp.saude.sc.gov.br/sistemas/revista/index.php/inicio/article/viewArticle/98.

22. Nussbaumer L, Dapper V. Investigação de óbitos por mesotelioma no Rio Grande do Sul, 1999 a 2003. Bol. Epidemiológico. [Internet] 2007;9(1) [acesso em 30 nov 2016]. Disponível: www.abrea.com.br/mesoteliomargs. pdf.

23. Pinto IV, Ramos DN, da Costa MCE, Ferreira CBT, Rebelo MS. Completude e consistência dos dados dos registros hospitalares de câncer no Brasil. Cad. Saúde Colet. [Internet] 2012;20(1) [acesso em 30 nov 2016]. Disponível: www.iesc.ufrj.br/cadernos/images/csc/2012_1/artigos/CSC_v20n1_113-120.pdf. 